\title{
High plant diversity is needed to maintain ecosystem services
}

Forest Isbell ${ }^{1}$, Vincent Calcagno ${ }^{1}$, Andy Hector ${ }^{2}$, John Connolly ${ }^{3}$, W. Stanley Harpole ${ }^{4}$, Peter B. Reich ${ }^{5,6}$, Michael Scherer-Lorenzen ${ }^{7}$, Bernhard Schmid $^{2}$, David Tilman ${ }^{8}$, Jasper van Ruijven ${ }^{9}$, Alexandra Weigelt ${ }^{10}$, Brian J. Wilsey ${ }^{4}$, Erika S. Zavaleta ${ }^{11} \&$ Michel Loreau $^{1}$

Biodiversity is rapidly declining worldwide ${ }^{1}$, and there is consensus that this can decrease ecosystem functioning and services ${ }^{2-7}$. It remains unclear, though, whether few ${ }^{8}$ or many $^{9}$ of the species in an ecosystem are needed to sustain the provisioning of ecosystem services. It has been hypothesized that most species would promote ecosystem services if many times, places, functions and environmental changes were considered ${ }^{9}$; however, no previous study has considered all of these factors together. Here we show that $84 \%$ of the 147 grassland plant species studied in 17 biodiversity experiments promoted ecosystem functioning at least once. Different species promoted ecosystem functioning during different years, at different places, for different functions and under different environmental change scenarios. Furthermore, the species needed to provide one function during multiple years were not the same as those needed to provide multiple functions within one year. Our results indicate that even more species will be needed to maintain ecosystem functioning and services than previously suggested by studies that have either (1) considered only the number of species needed to promote one function under one set of environmental conditions, or (2) separately considered the importance of biodiversity for providing ecosystem functioning across multiple years ${ }^{10-14}$, places ${ }^{15,16}$, functions $s^{14,17,18}$ or environmental change scenarios $^{12,19-22}$. Therefore, although species may appear functionally redundant when one function is considered under one set of environmental conditions ${ }^{7}$, many species are needed to maintain multiple functions at multiple times and places in a changing world.

Arguments for biodiversity conservation are often based on ecosystem services, but it remains unclear whether few ${ }^{8}$ or many ${ }^{9}$ species are needed to maintain ecosystem services. Determining how many species provide ecosystem services will require a synthesis of several areas of biodiversity research (Fig. 1). Biodiversity-ecosystem functioning studies have often considered a single functional context and found that multiple, but not all, study species promoted ecosystem functioning ${ }^{5-7}$ (Fig. 1a). We define a functional context (henceforth context) as the measurement of one function, at one time and place, under one environmental change scenario. Several related biodiversity studies have explored whether more species promote ecosystem functioning when more than one context is considered. For example, biodiversity-ecosystem stability (that is, the invariability of productivity) studies have found that more species are needed to provide ecosystem functioning at larger spatio-temporal scales because different species promote productivity at different times ${ }^{10-14}$ (Fig. 1b) or places ${ }^{15,16}$. Biodiversity-ecosystem multifunctionality studies have found that more species are needed to provide multiple functions because different species promote different functions ${ }^{14,17,18}$ (Fig. 1c). Biodiversityglobal change studies have found that more species are needed to provide ecosystem functioning in a changing world because different species promote ecosystem functioning under different environmental change scenarios ${ }^{12}$. Here, for the first time to our knowledge, we consider all of these relationships together.

We included data from 17 grassland biodiversity experiments that considered multiple times, places, functions or environmental change scenarios (Supplementary Table 1 and Supplementary Data). To test whether different species promoted ecosystem functioning during different years, we included studies that planted replicate plots (same species compositions) during consecutive years ${ }^{23}$ or made repeated measurements of ecosystem functions across years ${ }^{17,19,21,22,24,25}$. To test whether different species promoted ecosystem functioning at different places, we included studies that planted replicate plots (same species compositions, with one exception ${ }^{25}$ ) at multiple sites across Europe ${ }^{26}$ or multiple spatial blocks within a site ${ }^{17,23,25}$. To test whether different

a Do multiple species promote ecosystem functioning within a particular context?

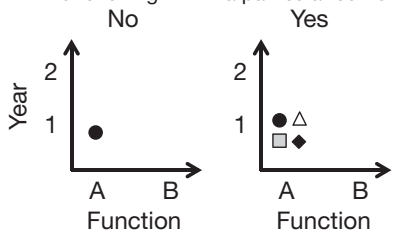

b Do different species promote ecosystem functioning during different years?

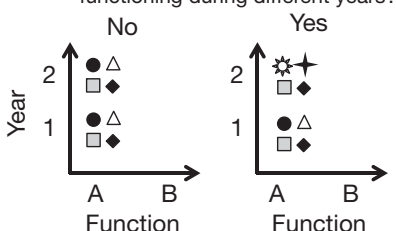

C Do different species promote different functions?

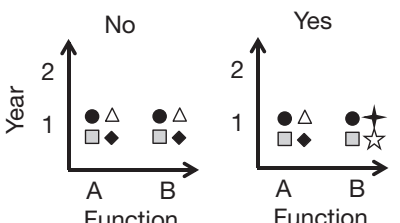

Do different species provide ecosystem stability and ecosystem multifunctionality?

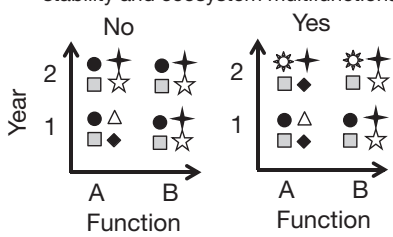

Species pool $\bullet \Delta \square-\frac{1}{4} \mathbf{Z}$

Figure 1 Some of the ways that biodiversity can be important for ecosystem functioning. Each of the eight symbols represents a species. Species shown in the bivariate plots are those that promoted ecosystem functioning within each functional context (for example, context A1 might be aboveground biomass measured during 2001). Although this figure defines contexts in two dimensions for simplicity, we considered four dimensions (Fig. 3). Previous studies have considered (a) one context, or $(\mathbf{b}, \mathbf{c})$ one dimension of contexts (b, ecosystem stability studies; c, ecosystem multifunctionality studies). Figure 2 tests the question in $\mathbf{d}$, by comparing the one-dimensional overlap (for example, between A1 and A2) with the two-dimensional overlap (for example, between A1 and B2) and three-dimensional overlap (that is, a pair of contexts that differs in three ways; not shown). Our results reject each of the null hypotheses shown on the left in $\mathbf{a}-\mathbf{d}$.

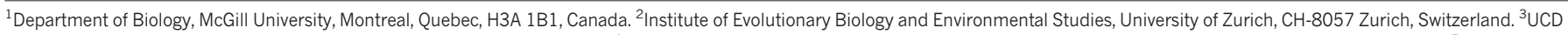

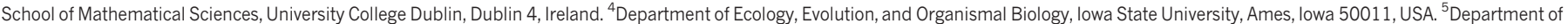

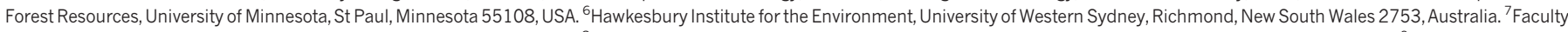

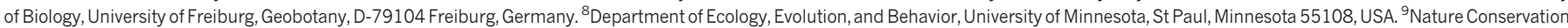

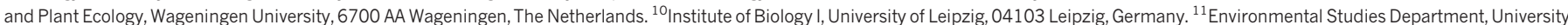
of California, Santa Cruz, California 95064, USA. 
species promoted different functions, we included studies that measured several functions ${ }^{14,17,22}$, such as biomass production and nutrient uptake. Many of these functions are considered to be supporting ecosystem services because other types of ecosystem services depend on them ${ }^{4,27}$. To test whether different species promoted ecosystem functioning under different environmental change scenarios, we included studies that applied environmental change treatments, such as nutrient and $\mathrm{CO}_{2}$ enrichment ${ }^{19}$, precipitation changes ${ }^{21}$ or land use changes such as livestock grazing ${ }^{22}$ and haying ${ }^{20}$.

We began by identifying the sets of study species that influenced ecosystem functioning within each context. Species were considered to promote ecosystem functioning in a particular context if they had effects in the direction that would usually be considered desirable from an ecosystem services perspective ${ }^{17}$. Positive effects were considered desirable for all functions except for soil inorganic nitrogen and light availability at ground level, where negative effects are consistent with lower levels of unconsumed resources ${ }^{17}$. We did not use separate definitions of desirable effects for different species (for example, positive effects of legumes on soil nitrogen might be considered desirable) to be consistent with previous studies ${ }^{17,18}$, to be conservative and because it may not be possible to manage simultaneously for both positive and negative effects. We found that approximately $27 \%$ of the study species promoted ecosystem functioning within any particular context, regardless of the size of the study species pool (Fig. 2a). Note that if many species were functionally redundant, or if only the most common species promoted ecosystem functioning, then we would expect a saturating relationship in Fig. 2a. Instead, our results suggest that even rare species can promote ecosystem functioning.

After identifying the sets of species that promoted ecosystem functioning in each context, we tested whether different sets of species promoted ecosystem functioning in different contexts. We used Sørensen's similarity index to quantify overlap between species sets ${ }^{17}$. All comparisons were made within studies so that differences between pairs of contexts were not due to sampling from multiple species pools. First, we quantified one-dimensional overlap between all pairs of contexts that differed in only one way (Fig. 1b, c). For example, multi-year overlap was quantified between each pair of contexts that differed only in which year ecosystem functioning was measured (that is, same place, function and environmental change scenario in both contexts) A multi-year overlap value of one or zero would respectively indicate that completely identical or completely unique sets of species promoted ecosystem functioning during different years, independent of the other sources of variation. We found overlap values between these two extremes, which indicates that somewhat different sets of species promoted ecosystem functioning during different years, at different places, for different functions and under different environmental change scenarios (Fig. 2b).

After considering these sources of variation independently, we quantified multi-dimensional overlap between pairs of contexts that differed in two or three ways (Fig. 1d). Again, all comparisons were made within studies. We found that the average overlap between pairs of contexts decreased as the number of differences between contexts increased (Fig. 2b and Supplementary Fig. 1). This means that, for example, the identities of the additional species needed to provide one function during multiple years were not the same as the identities of the additional species needed to provide multiple functions during one year (Fig. 1d). Additionally, species sets did not simply vary independently of context attributes (permutation test $P<0.001$ for one-, two- and three-dimensional overlap) (Fig. 2b). Thus, our results indicate that even more species will be needed to maintain ecosystem functioning and services than previously suggested by studies that have either (1) considered only the number of species needed to promote one function under one set of environmental conditions, or (2) separately considered the importance of biodiversity for providing ecosystem functioning across multiple years ${ }^{10-14}$, places ${ }^{15,16}$, functions ${ }^{14,17,18}$ or environmental change scenarios ${ }^{12,19-22}$. Future studies could more completely consider the consequences of biodiversity declines for ecosystem functioning and services by similarly considering the multidimensionality of ecosystem functioning both in experimental and natural communities.

Next, we quantified the extent to which the number of species promoting ecosystem functioning increased as more years, places, functions or environmental change scenarios were considered within each study. In other words, we quantified the accumulation of species across each of the four dimensions of contexts that we considered (Fig. 1). We found that a greater proportion of species promoted

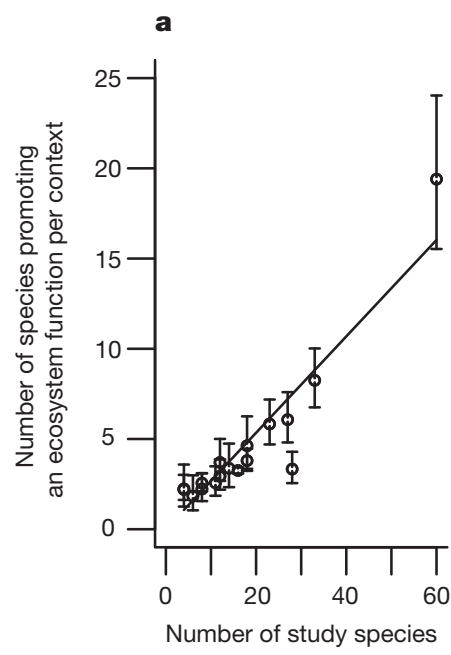

Figure $2 \mid$ Sets of study species that promoted ecosystem functioning. a, The mean number of species that promoted ecosystem functioning within each context increased linearly $\left(t=16.40, P<0.001, R^{2}=0.944\right)$ with the size of the species pool, such that approximately $27 \%$ (mean, $95 \%$ confidence intervals for slope: $0.27,0.24-0.30$ ) of the study species promoted ecosystem functioning within each context. Error bars for each study indicate $95 \%$ generalized linear model confidence intervals. b, Different sets of species promoted ecosystem

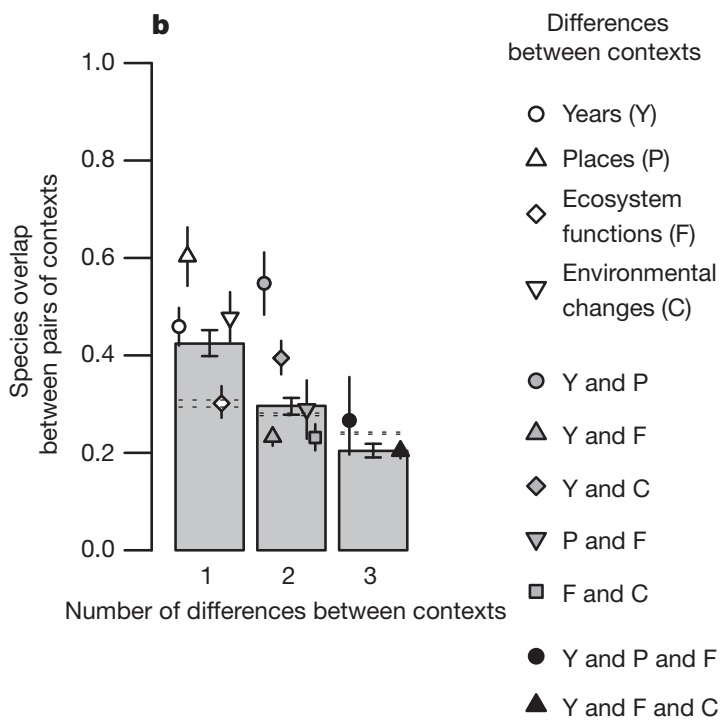

functioning in different contexts (overlap $<1$ ), and overlap between pairs of contexts decreased as the number of differences between contexts increased (see Fig. 1). Symbols indicate means for each specific type of overlap; horizontal dotted lines show $\pm 95 \%$ permutation test confidence intervals; error bars for symbols and bars indicate $95 \%$ bootstrap confidence intervals. Supplementary Data indicates numbers of contexts for each study. 
a

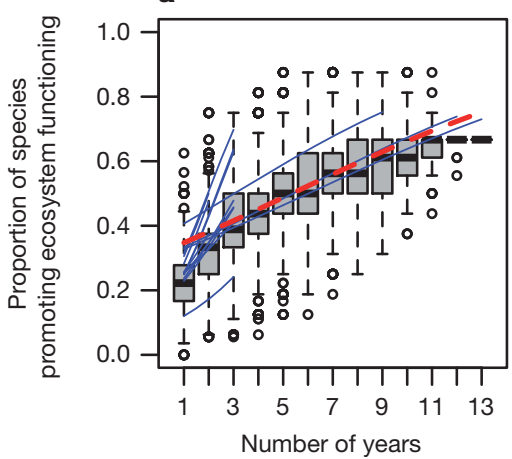

b

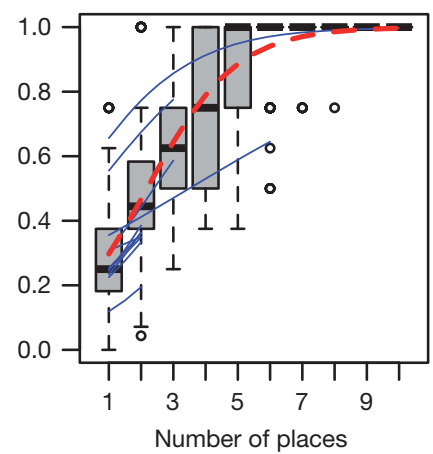

c

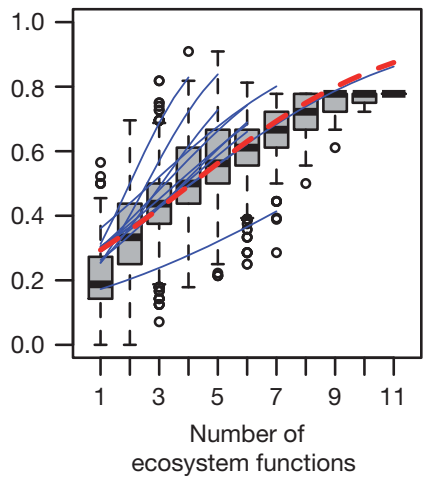

d

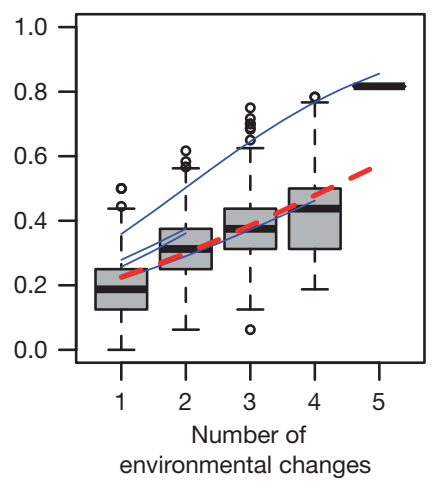

Figure 3 The proportion of study species that promoted ecosystem functioning increased when more (a) years, (b) places, (c) ecosystem functions and (d) environmental change scenarios were independently considered. Solid blue lines indicate generalized linear model fits for each study; dashed red line indicates grand mean generalized linear model fitted

ecosystem functioning when more years, places, functions or environmental change scenarios were considered (Fig. 3). These relationships result from different species promoting ecosystem functioning in different contexts (Fig. 2b). Note that if the one-dimensional overlap values corresponding to each panel in Fig. 3 were one or zero, then these relationships would be horizontal or linearly increasing, respectively ${ }^{17}$. Our results are between these two extremes.

After comparing contexts within studies, data from all studies were combined to consider how the total number of species that promoted ecosystem functioning increased with the total number of contexts. We quantified the number of species that promoted ecosystem functioning in a random subset of all possible combinations of our observed contexts (that is, 100 pairs, 100 groups of three, etc.). The large increase in the number of species that promoted ecosystem functioning as more contexts were considered (Fig. 4) is the result of different species promoting ecosystem functioning during different years, at different places, for different functions, under different environmental change scenarios and in different species pools. Considering all of these factors together suggests that many species will be needed to maintain ecosystem multifunctionality at large spatio-temporal scales in a changing world. Consequently, the extinction (or decreased local occurrence) of almost any of these species is expected to decrease ecosystem functioning and services in at least some contexts. Further study is needed to identify the processes that explain why different species promoted ecosystem functioning in different contexts. The specific mechanisms involved probably differed across contexts, but previous results from these and other biodiversity experiments ${ }^{28}$ suggest that complementarity (in time, space, functional effect traits and functional response traits) is a general explanation for this pattern.

Our results reveal new opportunities and challenges for prioritizing conservation efforts and predicting consequences of biodiversity declines. According to the precautionary principle, all species should be conserved because we cannot be certain which species actually provide ecosystem services ${ }^{29}$. Our results offer further support for the precautionary principle because most of the studied species were important at least once, and species exhibited context-dependent effects that will be difficult to predict. If it is impossible or impractical to conserve all species, then future studies could additionally consider how often (Supplementary Fig. 2 and Supplementary Data) and how much species influenced ecosystem functioning to determine which species are most important for maintaining ecosystem functioning and services. This will require careful consideration of many contexts, because it is not possible to make general predictions or conclusions by considering a few context-dependent phenomena ${ }^{30}$.

Future studies could determine whether some species consistently promote ecosystem functioning under environmental conditions that across all studies. Box plots summarize observed data: black band, median; bottom and top of boxes respectively correspond to lower and upper quartiles; error bars, \pm 1.5 times the interquartile range. See Supplementary Data for the specific years, places, functions and environmental change scenarios considered in each study.

are currently common, or under environmental change scenarios that will probably become increasingly common. Note that even species that have small effects could be important for maintaining ecosystem functioning and services if they have a large cumulative desirable effect across many contexts. For example, Eriochloa sericea had the smallest desirable effect on above-ground biomass in the irrigated plots at the MEND Irrigation experiment during 2009, but promoted ecosystem functioning in $75 \%$ of the contexts in which it was included. Future studies could also determine which species promote ecosystem functioning in particular contexts that are highly valued by stakeholders. Note that even species that rarely promote (or often decrease) ecosystem functioning could be most important for maintaining ecosystem functioning and services within some contexts. For example, although Pascopyrum smithii only promoted ecosystem functioning in $2 \%$ of the contexts in which it was included, it promoted soil carbon more than any other species in the Cedar Creek Biodiversity experiment during 2004. Furthermore, note that declines in local diversity, which are far more common than global extinctions, will also decrease ecosystem functioning and services within some contexts. Finally, even the few species that never promoted ecosystem functioning in these studies (Supplementary Fig. 2) could promote ecosystem functioning

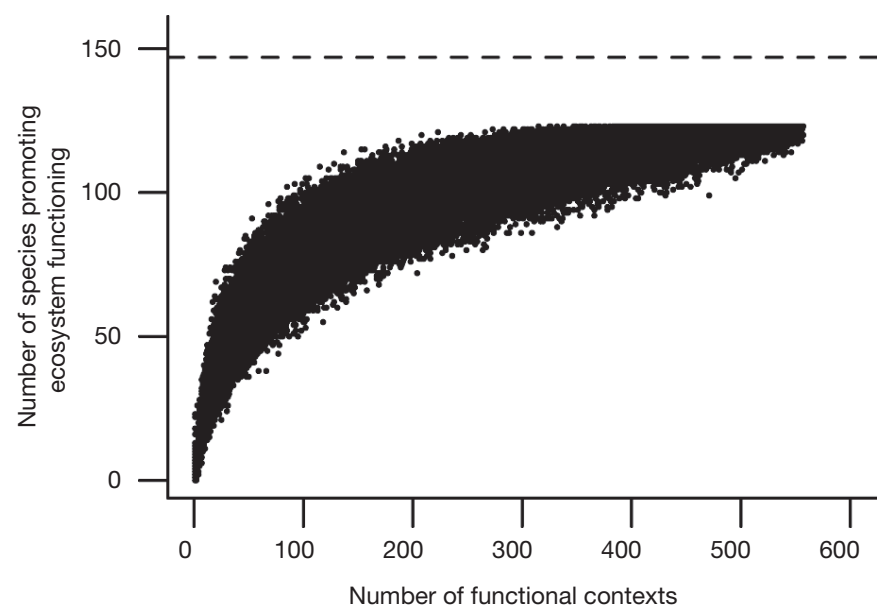

Figure 4 The number of study species that promoted ecosystem functioning increased with the number of contexts considered across all studies. The points are the number of species that promoted ecosystem functioning when 1-557 contexts were sampled from all 557 contexts. The dashed line indicates the total number of studied species (147), which restricts the upper limit for these values. The $x$ axis includes variation across years, places, functions, environmental change scenarios and species pools. 
in other contexts, or be a conservation priority for other (for example, ethical, aesthetic) reasons. Therefore, we encourage careful consideration of many contexts when making conservation decisions and predicting the consequences of biodiversity declines.

\section{METHODS SUMMARY}

Identifying the species that promoted ecosystem functioning. For each context, we modelled ecosystem functioning response variables as a function of the presence or absence of each study species at the plot level. We used backwardelimination multiple regression to identify a minimally adequate model, based on the Akaike information criterion (AIC) $)^{17}$. This procedure was performed with the stepAIC function in the MASS package of R 2.11.1 (see Methods). Species were considered to promote ecosystem functioning in a context if they were included in the minimally adequate model and had effects in the direction that would usually be considered desirable from an ecosystem services perspective ${ }^{17}$.

Comparing sets of species between pairs of contexts. We used Sørensen's similarity index to quantify the overlap between the sets of species that promoted ecosystem functioning in pairs of contexts within each study ${ }^{17}$. This allowed us to test whether identical (overlap $=1$ ), unique (overlap $=0$ ) or somewhat different $(0<$ overlap $<1)$ sets of species promoted ecosystem functioning in different contexts. We also tested whether overlap decreased as the number of differences between contexts increased (Fig. 1).

Accumulation of species across across contexts. We quantified the accumulation of species across each of the four dimensions of contexts that we considered (Methods; Fig. 1). A quasi-binomial generalized linear model was fitted to determine how the proportion of species that promoted ecosystem functioning increased with the number of years (or places, functions, environmental changes), including 'study' as a factor. This allowed us to describe the mean trends within and across studies. We also randomly sampled combinations (that is, 100 pairs, 100 groups of three, and so on) of all 557 contexts to determine how the number of species promoting ecosystem functioning increased when all of these factors were considered together.

Full Methods and any associated references are available in the online version of the paper at www.nature.com/nature.

Received 18 March; accepted 10 June 2011.

Published online 10 August 2011.

1. Butchart, S. H. M. et al. Global biodiversity: indicators of recent declines. Science 328, 1164-1168 (2010)

2. Loreau, M. et al. Biodiversity and ecosystem functioning: current knowledge and future challenges. Science 294, 804-808 (2001).

3. Hooper, D. U. etal. Effects of biodiversity on ecosystem functioning: a consensus of current knowledge. Ecol. Monogr. 75, 3-35 (2005).

4. Balvanera, P. et al. Quantifying the evidence for biodiversity effects on ecosystem functioning and services. Ecol. Lett. 9, 1146-1156 (2006).

5. Cardinale, B. J. etal. Effects of biodiversity on the functioning of trophic groups and ecosystems. Nature 443, 989-992 (2006)

6. Naeem, S., Bunker, D. E., Hector, A., Loreau, M. \& Perrings, C. Biodiversity, Ecosystem Functioning, and Human Wellbeing: An Ecological and Economic Perspective (Oxford Univ. Press, 2009)

7. Cardinale, B. J. et al. The functional role of producer diversity in ecosystems. Am. J. Bot. 98, 572-592 (2011).

8. Ridder, B. Questioning the ecosystem services argument for biodiversity conservation. Biodivers. Conserv. 17, 781-790 (2008).

9. Duffy, J. E. Why biodiversity is important to the functioning of real-world ecosystems. Front. Ecol. Environ 7, 437-444 (2009).

10. McNaughton, S. J. Diversity and stability of ecological communities: a comment on the role of empiricism in ecology. Am. Nat. 111, 515-525 (1977).

11. Yachi, S. \& Loreau, M. Biodiversity and ecosystem productivity in a fluctuating environment: the insurance hypothesis. Proc. Natl Acad. Sci. USA 96, 1463-1468 (1999).

12. Craine, J. M. et al. The role of plant species in biomass production and response to elevated $\mathrm{CO}_{2}$ and N. Ecol. Lett. 6, 623-630 (2003).

13. Tilman, D., Reich, P. B. \& Knops, J. M. H. Biodiversity and ecosystem stability in a decade-long grassland experiment. Nature 441, 629-632 (2006)
14. Zavaleta, E. S., Pasari, J. R., Hulvey, K. B. \& Tilman, G. D. Sustaining multiple ecosystem functions in grassland communities requires higher biodiversity. Proc. Natl Acad. Sci. USA 107, 1443-1446 (2010).

15. Loreau, M., Mouquet, N. \& Gonzalez, A. Biodiversity as spatial insurance in heterogeneous landscapes. Proc. Natl Acad. Sci. USA 100, 12765-12770 (2003).

16. Griffin, J. N. et al. Spatial heterogeneity increases the importance of species richness for an ecosystem process. Oikos 118, 1335-1342 (2009).

17. Hector, A. \& Bagchi, R. Biodiversity and ecosystem multifunctionality. Nature $\mathbf{4 4 8 ,}$ 188-190 (2007)

18. Gamfeldt, L., Hillebrand, H. \& Jonsson, P. R. Multiple functions increase the importance of biodiversity for overall ecosystem functioning. Ecology 89, 1223-1231 (2008).

19. Reich, P. B. et al. Plant diversity enhances ecosystem responses to elevated $\mathrm{CO}_{2}$ and nitrogen deposition. Nature 410, 809-812 (2001).

20. Weigelt, A., Weisser, W. W., Buchmann, N. \& Scherer-Lorenzen, M. Biodiversity for multifunctional grasslands: equal productivity in high-diversity low-input and lowdiversity high-input systems. Biogeosciences 6, 1695-1706 (2009).

21. Wilsey, B. J., Teaschner, T. B., Daneshgar, P.P., Isbell, F.I.\& Polley, H. W. Biodiversity maintenance mechanisms differ between native and novel exotic-dominated communities. Ecol. Lett. 12, 432-442 (2009).

22. Isbell, F. I. \& Wilsey, B. J. Increasing native, but not exotic, biodiversity increases aboveground productivity in ungrazed and intensely grazed grasslands. Oecologia 165, 771-781 (2011).

23. Isbell, F. I., Losure, D. A., Yurkonis, K. A. \& Wilsey, B. J. Diversity-productivity relationships in two ecologically realistic rarity and extinction scenarios. Oikos 117, 996-1005 (2008).

24. Tilman, D. et al. Diversity and productivity in a long-term grassland experiment Science 294, 843-845 (2001).

25. van Ruijven, J. \& Berendse, F. Long-term persistence of a positive plant diversityproductivity relationship in the absence of legumes. Oikos 118, 101-106 (2009).

26. Kirwan, L. et al. Evenness drives consistent diversity effects in intensive grassland systems across 28 European sites. J. Ecol. 95, 530-539 (2007).

27. Millennium Ecosystem Assessment. Ecosystems and Human Well-being: Synthesis (Island Press, 2005).

28. Cardinale, B. J. et al. Impacts of plant diversity on biomass production increase through time because of species complementarity. Proc. Natl Acad. Sci. USA 104, 18123-18128 (2007).

29. Ehrlich, P. \& Ehrlich, A. Extinction: The Causes and Consequences of the Disappearance of Species (Victor Gollancz, 1982)

30. Lawton, J. H. Are there general laws in ecology? Oikos 84, 177-192 (1999).

Supplementary Information is linked to the online version of the paper at www.nature.com/nature.

Acknowledgements We thank J. Byrnes, L. Gamfeldt and M. Emmerson for comments on an earlier version of this manuscript. We thank the Swiss SystemsX.ch initiative (IPP-2008/23) for supporting this project. The BIODEPTH project was funded by the European Commission within the Framework IV Environment and Climate Programme (ENV-CT95-0008) and by the Swiss Federal Office for Education and Science (Project EU-1311). The Jena Experiment was funded by the Deutsche Forschungsgemeinschaft (DFG, FOR 456), Friedrich Schiller University of Jena, Max Planck Society, University of Zurich, Swiss National Science Foundation (3100AO-107531) and ETH Zurich. The Wageningen experiment was funded by the Dutch Organisation for Scientific Research (NWO) within the framework of the Biodiversity Programme. Work on the Agrodiversity experiment was funded by the EU Commission through COST Action 852 and Science Foundation Ireland (09/RFP/EOB2546). The BioCON experiment was funded by the US Department of Energy (DOE/DE-FG02-96ER62291) and the US National Science Foundation (Biocomplexity 0322057, LTER DEB 9411972, DEB 0080382, DEB 0620652 and LTREB DEB 0716587). The MEND Irrigation, BioGEN and RarityExtinction experiments were funded by the US National Science Foundation (DEB 0639417). The Cedar Creek Biodiversity experiment was funded by the US National Science Foundation. M.L. was supported by The Natural Sciences and Engineering Research Council of Canada (Discovery Grant) and the Canada Research Chair program.

Author Contributions F.I. conceived the project; J.C., A.H., F.I., P.B.R., M.S.-L., B.S., D.T., J.v.R., A.W. and B.J.W. designed and conducted experiments; F.I. and V.C. analysed the data, with input from A.H. and M.L.; F.I. wrote the paper with input from all authors.

Author Information Reprints and permissions information is available at www.nature.com/reprints. The authors declare no competing financial interests. Readers are welcome to comment on the online version of this article at www.nature.com/nature. Correspondence and requests for materials should be addressed to F.I. (forest.isbell@gmail.com). 


\section{METHODS}

Identifying the species that promoted ecosystem functioning. For each context, we modelled ecosystem functioning response variables as a function of the presence or absence of each study species at the plot level. We used a backwardelimination multiple regression analysis to identify a minimally adequate model, based on the $\mathrm{AIC}^{17}$. This procedure was performed with the stepAIC function in the MASS package of R 2.11.1. Specifically, for each context, we started with a full model that included a main effect for each study species and an intercept. The stepAIC function then removed each species, one at a time, from this full model and compared the AIC values of the resulting simpler models with the AIC value of the full model. If the AIC value for any of the simpler models was smaller than the AIC value for the full model, then the variable whose removal resulted in the largest decrease in AIC was permanently removed from the full model. This backward-deletion process was repeated until the removal of any species resulted in a model with a higher AIC value. The minimally adequate model that resulted from this process contained the most parsimonious set of species influencing ecosystem functioning. These species were considered to promote ecosystem functioning if they had effects in the direction that would usually be considered desirable from an ecosystem services perspective ${ }^{17}$. Positive effects were considered desirable for all functions except for soil inorganic nitrogen and light availability at ground level, where negative effects are consistent with lower levels of unconsumed resources ${ }^{17}$. We did not use separate definitions of desirable effects for different species (for example, positive effects of legumes on soil nitrogen might be considered desirable) to be consistent with previous studies ${ }^{17,18}$, to be conservative and because it may not be possible to manage simultaneously for both positive and negative effects.

This modelling approach is conservative in several ways. Previous approaches for determining the number of species that promoted ecosystem functioning could have been biased by not allowing species to decrease ecosystem functioning ${ }^{18}$ or by not identifying which species actually influenced ecosystem functioning ${ }^{14}$. Furthermore, including species interactions in these or other models would probably increase the proportion of study species that promoted ecosystem functioning within each context ${ }^{12,26}$. For example, some species perform poorly in monocultures, but interact positively with other species in mixtures (for example, grass-legume interactions) ${ }^{31}$. Our approach would underestimate the desirable effects of these species. Also, the presence or absence model that we used would be especially conservative for experimental designs that concentrate on varying evenness rather than richness, such as the simplex design used in the Agrodiversity study ${ }^{26}$. Additionally, our results were qualitatively similar, but less conservative, when we used a model-averaging approach implemented in the glmulti package (version 0.6-3) of $\mathrm{R}^{32}$.

To compare the number of species that promoted ecosystem functioning across studies, we used a quasi-Poisson generalized linear model with 'study' as a main effect. This procedure was performed with the generalized linear model function in the stats package of R. The quasi-maximum-likelihood version of the Poisson generalized linear model accounts for over- or under-dispersion in the data. We used the confint.glm function in the MASS package of $\mathrm{R}$ to obtain the $95 \%$ confidence intervals. To quantify the proportion of study species that promoted ecosystem functioning, we regressed the mean number of species that promoted ecosystem functioning per context on the number of study species (that is, the number of planted species that were present at least once in biomass samples) with no intercept.

Comparing sets of species between pairs of contexts. After identifying the sets of species that promoted ecosystem functioning in each context, we quantified the overlap between these sets of species to test whether different sets of species promoted ecosystem functioning in different contexts. All overlap comparisons were made within studies. Overlap between contexts $a$ and $b$ was quantified by Sørensen's similarity index ${ }^{17}$ :

$$
o=\frac{\left|E_{a} \bigcap E_{b}\right|}{0.5\left(\left|E_{a}\right|+\left|E_{b}\right|\right)}
$$

where $\left|E_{a}\right|$ is the number of species that promoted ecosystem functioning in context $a$ and $\left|E_{a} \cap E_{b}\right|$ is the number of species that promoted ecosystem functioning in both contexts. First, one-dimensional overlap was quantified between pairs of contexts that only differed in one way (Fig. 1b, c). For example, multi-year overlap was quantified between each pair of contexts that differed only in which year ecosystem functioning was measured (that is, place, function and environmental change scenarios were the same in both contexts). This allowed us to test whether identical (overlap $=1)$, unique $($ overlap $=0$ ) or somewhat different $(0<$ overlap $<1)$ sets of species promoted ecosystem functioning in different contexts. Next, multi-dimensional overlap was quantified between pairs of contexts that differed in two or three ways (Fig. 1d). This allowed us to test whether overlap decreased as the number of differences between contexts increased (Fig. 1).

For each type of overlap, we used non-parametric bootstrap, with correction for bias $^{33,34}$, to build $95 \%$ confidence intervals. We re-sampled the observed contexts with replacement to generate each of 1,000 bootstrap data sets. For each of these bootstrap data sets, we computed the average overlap across all pairs of contexts that were relevant (that is, for the particular type of overlap being considered). We used this bootstrap approach to control for the non-independence of pairwise comparisons that had one context in common. We then used an exact permutation approach ${ }^{34}$ to test whether the observed overlap values differed from the expected null value (the null hypothesis being that overlap varied across contexts independent of context attributes: year, place, function and environmental change scenario). Within each study, we permuted context data (that is, the sequence of ones or zeros indicating whether each species had a desirable effect or not) with respect to context attributes. For each of 1,000 permutations, we computed the average overlap across all pairs of contexts that were relevant (that is, for the particular type of overlap being considered). We used the null distribution of overlap obtained from these random permutations to test whether the observed value was significantly lower (that is, in the lowest 2.5 percentiles) or significantly higher (that is, in the top 2.5 percentiles) than the null. We used this test because it does not assume that study species had independent responses to context attributes, and thus it should be robust to the presence of functional groups.

Note that there could be correlations between contexts owing to repeated measurements across years. These correlations could influence overlap estimates and tests that assume independent observations. For example, positive correlations between repeated measurements during consecutive years could lead to (1) overestimates of multi-year (Y) overlap, and (2) smaller estimates of overlap between two different years at two different places than between two different years at one place (that is, $\mathrm{Y}$ and places (P) overlap less than $\mathrm{Y}$ overlap). These potential correlations had little influence on our results because multi-year overlap estimates were much less than 1 , and $\mathrm{Y}$ and $\mathrm{P}$ overlap estimates were not less than $\mathrm{Y}$ overlap estimates (Fig. 2). Nevertheless, we encourage future studies to consider these correlations when interpreting overlap estimates.

Accumulation of species across contexts. We quantified the accumulation of species across each of the four dimensions of contexts that we considered (Fig. 1). For example, above-ground biomass was sampled during 13 years at the Cedar Creek Biodiversity experiment. To determine the extent to which more species promoted above-ground biomass as more years were considered, we sampled all combinations of these 13 contexts (that is, all pairs, groups of three, etc.), and recorded the number of unique species that promoted ecosystem functioning, and the number of unique years, for each combination. This was repeated for each function, at each place, under each environmental change scenario. These results are summarized in Fig. 3a. A quasi-binomial generalized linear model was fitted to determine how the proportion of species that promoted ecosystem functioning increased with the number of years, including 'study' as a factor. This allowed us to describe the mean trends within and across studies. We also randomly sampled combinations (that is, 100 pairs, 100 groups of three, etc.) of all 557 contexts to determine how the number of species promoting ecosystem functioning increased when all of these factors were considered together.

31. Nyfeler, D. etal. Strong mixture effects among four species in fertilized agricultura grassland led to persistent and consistent transgressive overyielding. J. Appl. Ecol. 46, 683-691 (2009).

32. Calcagno, V. \& de Mazancourt, C. glmulti: an R package for easy automated mode selection with (generalized) linear models. J. Stat. Softw. 34, 1-29 (2010).

33. Efron, B. \& Tibshirani, R. Bootstrap methods for standard errors, confidence intervals, and other measures of statistical accuracy. Stat. Sci. 1, 54-77 (1986).

34. Manly, B. F. J. Randomization, Bootstrap and Monte Carlo Methods in Biology 2nd edn (Chapman and Hall, 1997). 